Impulse geben für die Weiterentwicklung der Qualität im Gesundheitswesen, dafür steht der Swiss Quality Award. Deshalb finden Sie auf www.swissqualityaward.ch $\rightarrow$ Die Projekte eine reiche Auswahl überzeugender Qualitätsprojekte im Gesundheitswesen, welche sich in der Vergangenheit um den Swiss Quality Award beworben haben. Vielleicht inspiriert Sie eines dieser Projekte zu einer eigenen Umsetzung? Oder Sie erschliessen sich einen neuen Kontakt für den fachlichen Austausch? Die zahlreichen Projekteinreichungen in den vergangenen Jahren zeigen, dass Qualität in der täglichen Arbeit im Gesundheitswesen auf vielfältige Weise gelebt wird. Nutzen auch Sie die Chance und zeigen Sie auf, wie Ihr innovatives Projekt zur Weiterentwicklung der Qualität im Gesundheitswesen beiträgt: Bewerben Sie sich bis am 8. Januar 2016 für den Swiss Quality Award!

Dr. med. Christoph Bosshard, Mitglied des Zentralvorstandes der FMH, Departementsverantwortlicher Daten, Demographie und Qualität / Schweizerische Akademie für Qualität in der Medizin SAQM

\title{
Swiss Quality Award 2016 auf Gewinnersuche
}

\section{Michelle Gerber}

lic. phil. hum., wissenschaftliche Mitarbeiterin FMH, Abteilung Daten, Demographie und Qualität DDQ / Schweizerische Akademie für Qualität in der Medizin SAQM

\author{
Jetzt ist es wieder so weit: Ab sofort können Sie sich mit Ihrem Qualitätsprojekt um \\ den Swiss Quality Award 2016 bewerben! Gemeinsam mit der SQMH und dem IEFM \\ verleiht die FMH/SAQM diesen Preis zur Anerkennung der Arbeit von Pionierinnen \\ und Pionieren, welche die Qualität im Gesundheitswesen voranbringen.
}

\begin{abstract}
Der Swiss Quality Award zeigte über die letzten Jahre durch seine konstant hohe Teilnehmerzahl, dass im Gesundheitswesen unermüdlich und mit grossem Einsatz für höhere Qualität gearbeitet wird. Um den vergangenen Swiss Quality Award haben sich insgesamt 67 Projektteams beworben. Auch 2016 wollen die drei Trägerorganisationen, die Schweizerische Gesellschaft für Qualitätsmanagement im Gesundheitswesen SQMH, das Institut für Evaluative Forschung in der Medizin der Universität Bern IEFM sowie die Verbindung der Schweizer Ärztinnen und Ärzte FMH, mit dem Swiss Quality Award vorbildlichen Qualitätsprojekten zur verdienten Beachtung verhelfen. Dabei können nicht nur die Gewinner ihre Projekte auf der Webseite des Swiss Quality Award einem breiten Publikum präsentieren, sondern auch weitere überzeugende Qualitätsprojekte. So wird ein umfangreiches, praxisrelevantes Qualitätswissen öffentlich, belebt Fachdiskussionen und fördert die Vernetzung. Davon profitieren nicht nur interessierte Gesundheitsfachleute und -institutionen, sondern letztlich auch die Patientinnen und Patienten.
\end{abstract}

Der Swiss Quality Award wird in den drei Kategorien "stationärer Sektor», "ambulanter Sektor» und «sektorübergreifend» verliehen. Jede Preiskategorie ist mit 10000 Franken dotiert. Zusätzlich vergibt die Jury unter den besten eingereichten Projekten den «Swiss Quality Poster-Award» im Wert von 2000 Franken. Ob in der ambulanten Praxis oder der Hauspflege, in der Klinik oder im Pflegeheim - Qualitätsprojekte können in allen Bereichen viel bewirken. Bewerben können sich die unterschiedlichsten Projekte, unabhängig davon, auf welche Weise eine Qualitätsverbesserung im Gesundheitswesen erreicht wird. Auch kleinere Projekte haben grosse Gewinnchancen, wenn sie eine nachhaltige Verbesserung für Patientinnen und Patienten bewirken. Denn so können auch sie zur Inspiration für andere Organisationen werden.

Hat sich in Ihrer Praxis, Klinik, Organisation oder Ihrem Pflegeheim eine Idee bewährt und Sie möchten diese einem grossem Publikum vorstellen? Dann reichen Sie Ihr Projekt jetzt für den Swiss Quality Award 2016 ein! Entscheidend ist nicht der Umfang, sondern die Wirksamkeit Ihrer Arbeit! Anmelden können Sie sich online unter www.swissqualityaward.ch $\rightarrow$ Die Anmeldung. Anmeldeschluss ist der 8. Januar 2016.

Der Swiss Quality Award wird anlässlich des Nationalen Symposiums für Qualitätsmanagement im Gesundheitswesen am 1. Juni 2016 in Solothurn verliehen. Weitere Informationen zum Swiss Quality Award finden Sie auf www.swissqualityaward.ch
JETZT PROJEKTE EINREICHEN! BIS 08.01.2016

Der Swiss Quality Award prämiert herausragende Qualitätsinnovatione auf www.swissqualityaward.ch an. 